\title{
Are bioactive-rich fractions functionally richer?
}

\begin{abstract}
Plant bioresources are relied upon as natural, inexpensive, and sustainable remedies for the management of several chronic diseases worldwide. Plants have historically been consumed for medicinal purposes based on traditional belief, but this trend is currently changing. The growing interest in the medicinal properties of plant bioresources stems from concerns of side effects and other adverse effects caused by synthetic drugs. This interest has yielded a better understanding of the roles of plant bioactive compounds in health promotion and disease prevention, including the underlying mechanisms involved in such functional effects. The desire to maximize the potential of phytochemicals has led to the development of "rich fractions," in which extracts contain bioactive compounds in addition to elevated levels of the primary compound. Although a rich fraction effectively increases the bioactivity of the extract, the standardization and quality assurance process can be challenging. However, the supercritical fluid extraction (SFE) system is a promising green technology in this regard. Future clinical and pharmacological studies are needed to fully elucidate the implications of these preparations in the management of human diseases, thereby fostering a move toward evidence-based medicine.
\end{abstract}

Keyword: Bioactives; Chronic disease; Medicinal plants; Phytochemicals; Rich fraction 